\title{
Electrocardiographic Findings in Acutely and Chronically T. cruzi-infected Mice Treated by a Phenyl-Substituted Analogue of Furamidine DB569
}

\author{
Elen M. de Souza, Gabriel M. Oliveira and Maria de Nazaré C. Soeiro \\ Lab. Biologia Celular, DUBC, Instituto Oswaldo Cruz, FIOCRUZ, Rio de Janeiro, RJ, Brasil.
}

\begin{abstract}
Aromatic diamidines have been successfully used to combat a wide range of parasites that cause important human infections. Recently we reported that a $N$-phenyl-substituted analogue of furamidine (DB569) exerts a micromolar trypanocidal activity against Trypanosoma cruzi in vitro. Since DB569 also reduces the cardiac parasitism and increases the survival rates of T. cruzi-infected mice, our present aim was to analyze the potential protection of DB569 in the development of altered cardiac electrical conduction system during acute and chronic $T$. cruzi infection. In our experimental model of acute infection (Swiss mice inoculated with Y strain of T. cruzi), the prevailing disorder observed in electrocardiogram (ECG) analyses was sinus bradycardia. This ECG alteration was reverted in acutely infected mice treated with DB569. Interestingly, the DB569 treatment reduced significantly the numbers of CD 8 T cells in the cardiac infiltration. In addition, the noticed protection of DB569 in the ECG findings of acutely-infected animals was further extended to the chronic infection. Our data suggest that the reversion to and further maintenance of normal ECG profile in the DB569treated infected animals may be associated with the reduced cardiac CD $8^{+}$lymphocyte infiltration and parasitism that might be ultimately contributing to their increased survival rates.
\end{abstract}

Keywords: electrocardiography, aromatic diamidines, therapy, infected mice, inflammation, heart, Chagas' disease.

\section{Introduction}

Trypanosoma cruzi is the ethiological agent of Chagas' disease, which affects over 16-18 million people in endemic areas of South and Central America, leading to 50,000 deaths per year (WHO 2002). The disease has two phases: the acute, which appears shortly after the infection, and the chronic phase, which can develop in about one-third of the infected individuals after a silent period of years or decades called the indeterminate phase (Cunha-Neto et al. 2006).

Although the main clinical manifestations of the Chagas disease include both the cardiac and the digestive alterations, the former is the most prominent: about $25 \%$ to $30 \%$ of the infected people will develop a progressive heart disease commonly displaying symptomatic ventricular arrhythmias, hypertrophy; congestive heart failure; sinus bradycardia and tachycardia; thomboembolism and sudden death (Marin-Neto et al. 1999; Salles et al. 2003). Several hypotheses have been raised to explain the chronic chagasic cardiomyopathy (CCC) including parasite persistence (Golgher and Gazzinelli, 2004), microvascular dysfunction (Petkova et al. 2001), and imbalanced immune response with autoimmune implications (Leon and Engman, 2003).

The sudden cardiac death is one of the most common terminal events in Chagas' disease with these patients usually presenting complex and sustained ventricular arrhythmia on ambulatory electrocardiogram (ECG) (Maguire et al. 1987). The most frequent chagasic electrocardiographic abnormalities include (i) frequent sinus node dysfunction, (ii) high rate of involvement of the conduction system producing ECG alterations of the right bundle branch or left anterior hemiblock or both, (iii) high rate of ventricular arrhythmias, (iv) high frequency of atrioventricular block, (v) abnormal Q waves, STsegment and T-waves and (vi) autonomic dysfunction (Salles et al. 2003; Garcia et al. 2005). It has been reported that most patients that present CCC display altered ECG (Rocha et al. 2003). In a recent study it has been demonstrated that patients with positive serology for T. cruzi and active infection, but with normal left ventricular cineangiography, showed 100\% survival at 16 years follow-up, while the

Correspondence: Maria de Nazaré C. Soeiro. Tel: 005521 2598-4331; Fax: 005521 2260-4434; Email: soeiro@ioc.fiocruz.br

Please note that this article may not be used for commercial purposes. For further information please refer to the copyright statement at http://www.la-press.com/copyright.htm 
presence of apical aneurysm or left ventricular systolic dysfunction was along with $60 \%$ of mortality at 4 years follow-up (Dávila-Spinetti et al. 2005).

The available chemotherapy against T.cruzi based on nitrofurans (nifurtimox-Bayer) and nitroimidazoles (benznidazole-Roche) is unsatisfactory since both compounds are effective only against recent infections and have frequent toxic side effects besides the occurrence of drug resistance (reviewed in Coura and De Castro, 2002). Recent reviews clearly point to the need of finding more efficient and less toxic drugs. In this respect, aromatic diamidines represent a promising class of DNA-targeted anti-parasitic agents (reviewed in Soeiro et al. 2005; Wilson et al. 2005; Werbovetz, 2006). We have recently reported the high in vitro trypanocidal activity of DB569, a $N$-phenylsubstituted analogue of furamidine (De Souza et al. 2004). We also found that the treatment of acutely infected mice with DB569 significantly reduced the cardiac parasitism and partially increased the survival rates (De Souza et al. 2006a).

Since ECG alterations have also been reported in T.cruzi-infected mice (Bustamante et al. 2003, 2005; Zaidenberg et al. 2006) our aim in the present study was to evaluate the potential protection of DB569 in the development of cardiac alterations in both acutely and chronically $T$. cruzi infected mice.

\section{Materials and Methods}

\section{Parasites}

Bloodstream trypomastigotes of the Y strain were harvested by heart puncture from $T$. cruzi-infected Swiss mice at the parasitaemia peak, as described previously (Meirelles et al. 1982).

\section{In vivo infection}

Male Swiss mice (25-30 g) were obtained from the Fundação Oswaldo Cruz (FIOCRUZ) animal facilities (Rio de Janeiro, Brazil). Mice were housed at maximum 8 per cage and kept in a conventional room at $20-24{ }^{\circ} \mathrm{C}$ under a $12 / 12-\mathrm{h}$ light/dark cycle. The animals were provided with sterilized water and chow ad libitum. Infection was performed by intraperitoneal (i.p.) injection of $10^{4}$ bloodstream trypomastigotes, and age-matched uninfected mice were maintained under identical conditions. All assays were performed 3 to 4 times and all procedures were carried out in accordance with the guidelines established by the FIOCRUZ Committee of Ethics for the Use of Animals (CEUA 0099/01).

\section{Experimental groups}

The animals were divided into the following groups: uninfected (non-infected and non-treated); untreated (infected with T. cruzi but non-treated); and treated (infected and treated with $20 \mathrm{mg} / \mathrm{kg}$ DB569). For analyses in the acute infection, at least 6 mice from each group were used at each different day post infection (dpi) until the 21st dpi. After one-year post infection, the surviving T. cruziinfected animals were used as the chronic mice group.

\section{Drugs and treatment schedule}

The synthesis of DB569 has been reported previously (Lansiaux et al. 2002). Stock solution (200 $\mathrm{mg}$ ) of the diamidine was prepared in dimethylsulfoxide and mice received $0.1 \mathrm{~mL}$ i.p. injection of $20 \mathrm{mg} / \mathrm{kg} /$ day DB569, starting at 3 dpi for 10 consecutive days as described (De Souza et al. 2006a). The infected and untreated mice group only received $0.1 \mathrm{~mL}$ i.p. daily injection of phosphatebuffered saline (10 mM sodium phosphate, $0.015 \mathrm{M}$ $\mathrm{NaCl}, \mathrm{pH}$ 7.4) (PBS) as vehicle.

\section{Parasitaemia and mortality}

Parasitaemia was individually checked by direct microscopic counting of parasites in $5 \mu \mathrm{L}$ of blood, as described before (De Souza et al. 2006a). Mortality was checked daily until 30 dpi and expressed as percentage of cumulative mortality (\%CM) (De Souza et al. 2006a).

\section{Histopathological analysis}

At 8, 15, 21 dpi and after one year of infection, hearts were removed, cut longitudinally, rinsed in ice-cold PBS and fixed in Millonig-Rosman solution $(10 \%$ formaldehyde in phosphate-buffered saline). The tissues were then dehydrated and embedded in paraffin. Sections $(3 \mu \mathrm{m})$ stained by routine hematoxylin-eosin (HE) were analyzed by light microscopy. The number of amastigote nests and of inflammatory infiltrates (more than 10 mononuclear cells) was determined in at least 30 fields (total magnification, 40X) for each slide. The 
mean number of amastigotes' nests or inflammatory infiltrates per field was obtained from at least three mice per group with three sections from each mouse.

\section{Flow Cytometry analysis of cardiac $\mathrm{CD} 4^{+}$and $\mathrm{CD}^{+} \mathrm{T}$ cells}

To evaluate the levels of cardiac $\mathrm{CD} 8^{+}$and $\mathrm{CD} 4^{+}$ $T$ cells, three mice of each experimental group were sacrificed at the peak of cardiac inflammation (15 dpi) (De Souza et al. 2006a), the hearts quickly removed and their inflammatory cells isolated for flow cytometry analysis as reported, with minor modifications (Marino et al. 2004). Briefly, to isolate mononuclear cells invading the cardiac tissue, hearts were fragmented (1 to $2 \mathrm{~mm}$ ) and submitted to seven enzymatic dissociations employing $0.2 \%$ collagenase. The recovered cells were washed in PBS, fixed for 20 min with $1 \%$ parafomaldehyde and labeled with phycoerythrin (PE)-conjugated anti-CD4 and Cy-Chromeconjugated anti-CD8 mAb (Pharmingen/BD Biosciences, San Diego, CA). Negative controls for specific labeling were prepared from isotypematched mAbs (Pharmingen/BD Biosciences).

A total of 10,000 events were acquired using a FACSCalibur flow cytometer (Becton Dickinson, San Jose, CA, USA) equipped with Cell Quest software. The data analysis was performed in the WinMDI software (Multiple Document Interface Flow Cytometry Application, v2.8, by Joseph Trotter, Scripps Research Institute, San Diego, CA, USA).

\section{Electrocardiography}

ECG recording and analysis were performed in uninfected, acutely $(8,15,21 \mathrm{dpi})$ and chronically (after 1 year post infection) T. cruzi-infected mice submitted or not to the DB569 therapy, as reported (Garcia et al. 2005; Zeidenberg et al. 2006). Briefly, mice were placed under stable sedation with diazepan $(20 \mathrm{mg} / \mathrm{kg}$, i.p.), fixed in the supine position, and eight-lead ECGs were recorded from 18-gauge needle electrodes subcutaneously implanted in each limb and two electrodes at precordial positions lead II. The electrocardiographic tracing was obtained with standard lead (dipolar lead DII), recording with amplitude set to give $2 \mathrm{mV} / 1 \mathrm{~s}$. ECGs were recorded by using a band-pass filtering (Bio Amp - AD Instruments, Hastings, United Kingdom) between 0.1 and $100 \mathrm{~Hz}$. Supplementary amplification and analog-digital conversion was performed with a Powerlab $16 \mathrm{~S}$ instrument (AD Instruments, Hastings). Digital recordings (16 bit, $4 \mathrm{kHz} /$ channel) were analyzed with the Scope (version v3.6.10) program (AD Instruments). The signal-averaged ECG (SAECG) was calculated by using the mouse SAECG extension (version 1.2) program (AD Instruments) and a templatematching algorithm. ECG parameters were evaluated in the acute and chronic chagasic mice by using the following standard criteria: (i) the heart rate was monitored by beats/minute (bpm), and (ii) the variation at $\mathrm{P}$ wave and $\mathrm{PQ}, \mathrm{QRS}$ and $\mathrm{QT}$ intervals were measured at milliseconds (ms).

\section{Statistical analysis}

Statistical analysis was carried out using the variance (ANOVA), with the level of significance set at $p \leq 0.05$. The data are representative of $2-4$ experiments run in duplicate.

\section{Results}

DB569 (Fig. 1) is effective towards both the intracellular and trypomastigote forms of $T$. cruzi, presenting $\mathrm{IC}_{50}$ values at low-micromolar doses in vitro (De Souza et al. 2004). This furamidine analogue also decreases mouse mortality and the levels of alanine aminotransferase and creatinine, indicating a protective role respectively against hepatic and renal lesions caused by the parasite infection (De Souza et al. 2006a). Since it significantly decreases cardiac parasitism to a level similar to that found for benznidazole treatment (De Souza et al. 2006a), we decided to investigate the potential protection of DB569 for the development of cardiac electrophysiologic abnormalities during acute and chronic T. cruzi infection. We confirmed our previous data showing that while the diamidine therapy slightly reduces the parasitaemia levels (Fig. 2A), DB569 significantly controls the cardiac parasitism, reducing in $95 \%$ the number of parasite nests $(p \leq 0.049)$ at the 15 th dpi (Fig. 2B and G), as compared to untreated animals (Fig. 2B and F). In addition, the flow cytometry analysis revealed that DB569 decreased in $40 \%$ the frequency of cardiac infiltrating $\mathrm{CD}^{+}$ $\mathrm{T}$ cells (Fig. 2E), as compared to the untreated animals (Fig. 2D). However, histological analysis of heart samples at the 15 th dpi, which represent the peak of both cardiac parasite load as well as inflammation in our experimental model (De Souza 


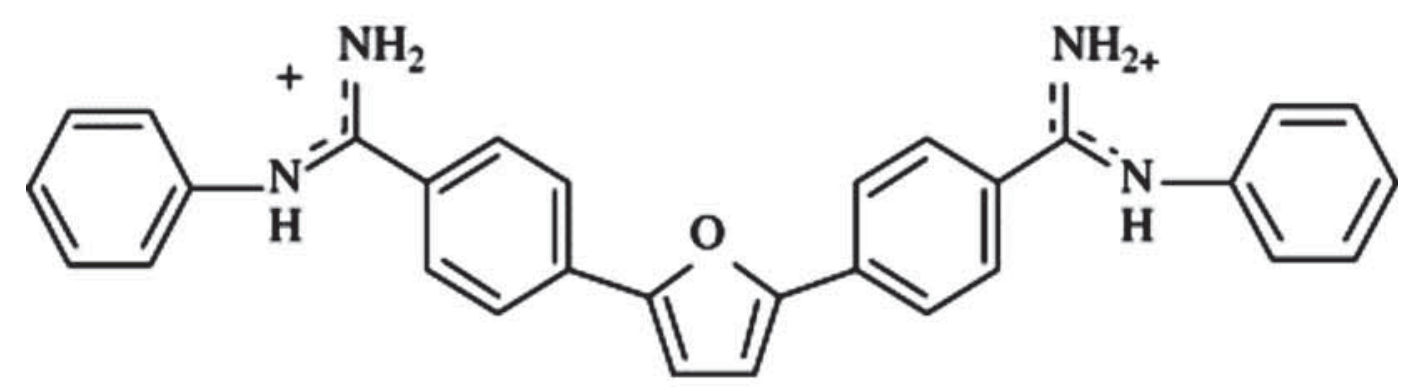

\section{DB569}

Figure 1. Structure of the DB569.

et al. 2006a), showed non-significant reduction $(p \leq 0.5)$ of the inflammation (Fig. 2C, H-I). Interestingly, the scarce parasite' nests found in heart tissues of drug-treated mice (Fig. 2G, asterisks) were frequently associated to inflammatory cells (Fig. 2G, arrow). Next we analyzed the electrocardiogram profiles in uninfected, untreated and DB569-treated mice (Fig. 3A). No differences among the groups were observed at the 8th dpi (Fig. 3A-B). However, at 15 and $21 \mathrm{dpi}$, we noticed sinus bradycardia as the prevailing ECG disorder found in untreated mice as compared to uninfected group, as detected by low heart rates (Fig. 3A,C-D). While uninfected mice exhibited values ranging from 630 to 670 beats/min (bpm), untreated and DB569-treated animals, displayed decreased heart frequencies, reaching 496 and 501 bpm, respectively (Fig. 3A and C). Interestingly, although decreased cardiac frequencies were maintained in the untreated mice at the 21st dpi, DB569treatment reverted $(p \leq 0.01)$ this ECG alteration, reaching values similar to the counterpartuninfected group (Fig. 3A and D). As diamidine treated group showed $40 \%$ reduction of the mortality compared to non-treated mice (Fig. 3E), we further investigated if the protection exerted by DB569 during the acute infection would be maintained in the chronic phase. We again observed lower cardiac frequency as the prevailing electrocardiographic change found in the chronic untreated group. We also found that DB569- treated mice reverted the cardiac alterations found in untreated animals $(p \leq 0.01)$, showing ECG profiles similar to uninfected mice and indicating that they sustained normal ECG profiles (Fig. 4A-B) as recovered at the 21 st dpi in the acutely-infected mice.

\section{Discussion}

The lack of an effective therapy for Chagas' disease associated to the toxicity and side effects of the available drugs (nifurtimox and beznidazole) justify the investigation of different natural as well as synthetic compounds that could substitute the current chemotherapy (Coura and de Castro, 2002). The well-known activity of aromatic diamidines towards many pathogens in both in vitro and in vivo studies (reviewed in Soeiro et al. 2005) prompted us to verify the effect of furamidine and its $N$-phenyl-substitute analogue, DB569. Moreover, the superior activity of DB569 as compared to furamidine even after short periods of in vitro treatment, without exerting toxicity towards mammalian cells (De Souza et al. 2004), impelled further analysis of the DB569 activity during T. cruzi infection in vivo (De Souza et al. 2006a). As electrocardiographic studies give important information regarding aspects of cardiac function, representing a useful tool to measure the severity and prognosis of the chagasic cardiopathy (Salles et al. 2003), here we sought to evaluate the potential protection of 

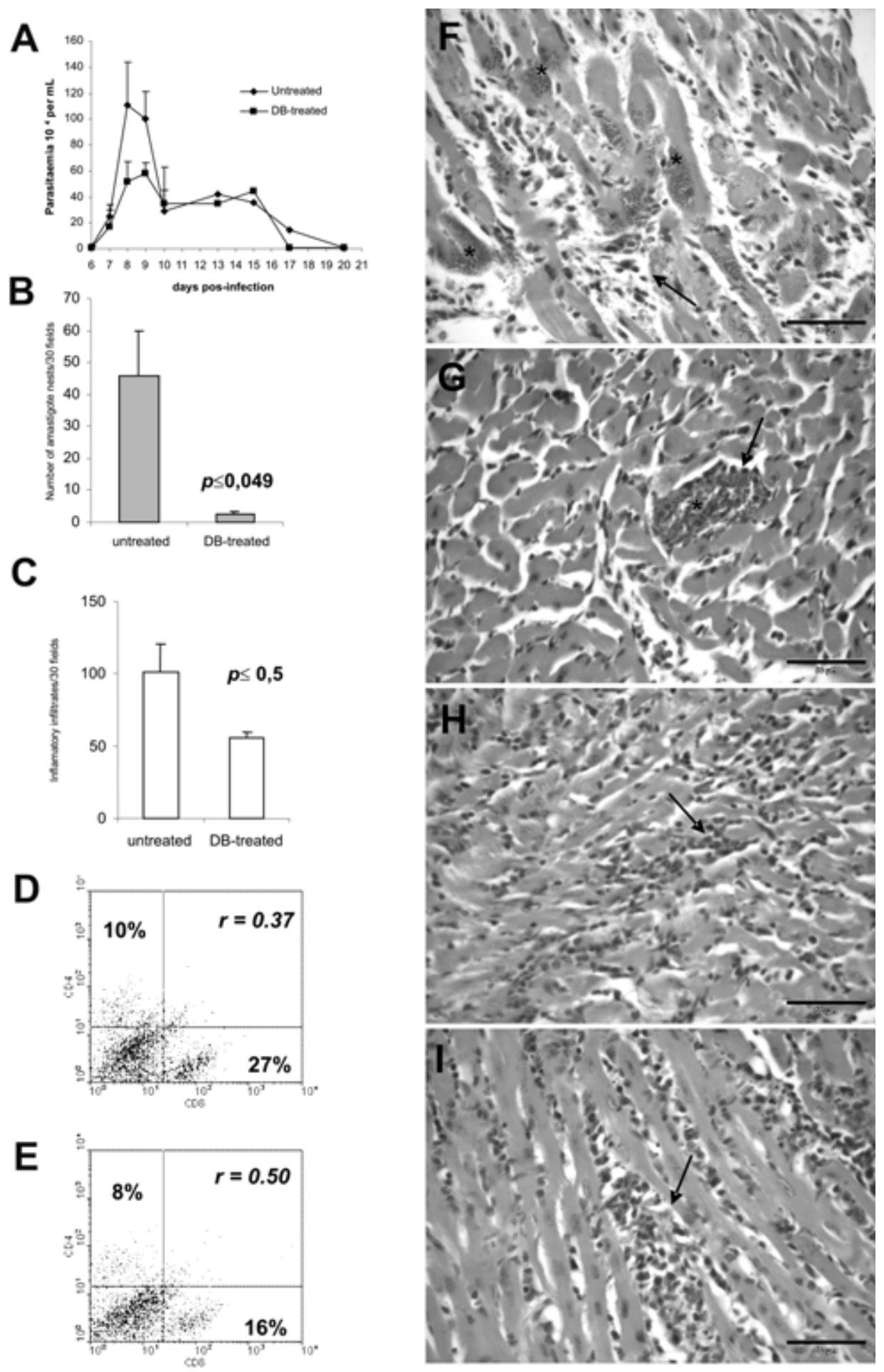

Figure 2. Treatment of T. cruzi-infected mice (104 Y strain/mice) with DB569 from 3 to 12 dpi: (A) Kinetics of parasitaemia, ( $\downarrow)$ Untreated (infected and non-treated) and (-) DB-treated (infected and treated with $20 \mathrm{mg} / \mathrm{kg} / \mathrm{day}$ DB569); (B) Number of amastigote' nests and (C) Inflamatory infiltration within the heart of untreated and DB-treated groups at 15 th $\mathrm{dpi}$; Cardiac CD $4^{+}$and CD $8^{+} \mathrm{T}$ cells expression measured by flow cytometry analysis of (D) untreated and (E) DB-treated T. cruzi-infected mice at 15th dpi. The lymphocyte gate was determined according to expression of $\mathrm{CD}^{+}$and $\mathrm{CD}^{+}$in $\mathrm{FSC} \times \mathrm{SSC}$ dot plot in infected animals; Histological patterns in heart sections at 15th dpi (F-I) of untreated $(\mathrm{F}$ and $\mathrm{H}$ ) and DB-treated ( $\mathrm{G}$ and $\mathrm{I}) \mathrm{T}$. cruzi-infected mice. Note the presence of parasite nests ( $\mathrm{F}$ and $\mathrm{G}$, asterisks) and the intensity of the inflammatory process (F-l, arrow). Bar $=50 \mu \mathrm{M}$. $p$ indicate significant differences between DB-treated and untreated groups. $r$ means the ratio between $\mathrm{CD} 4^{+}$and $\mathrm{CD} 8^{+}$cardiac cells). 


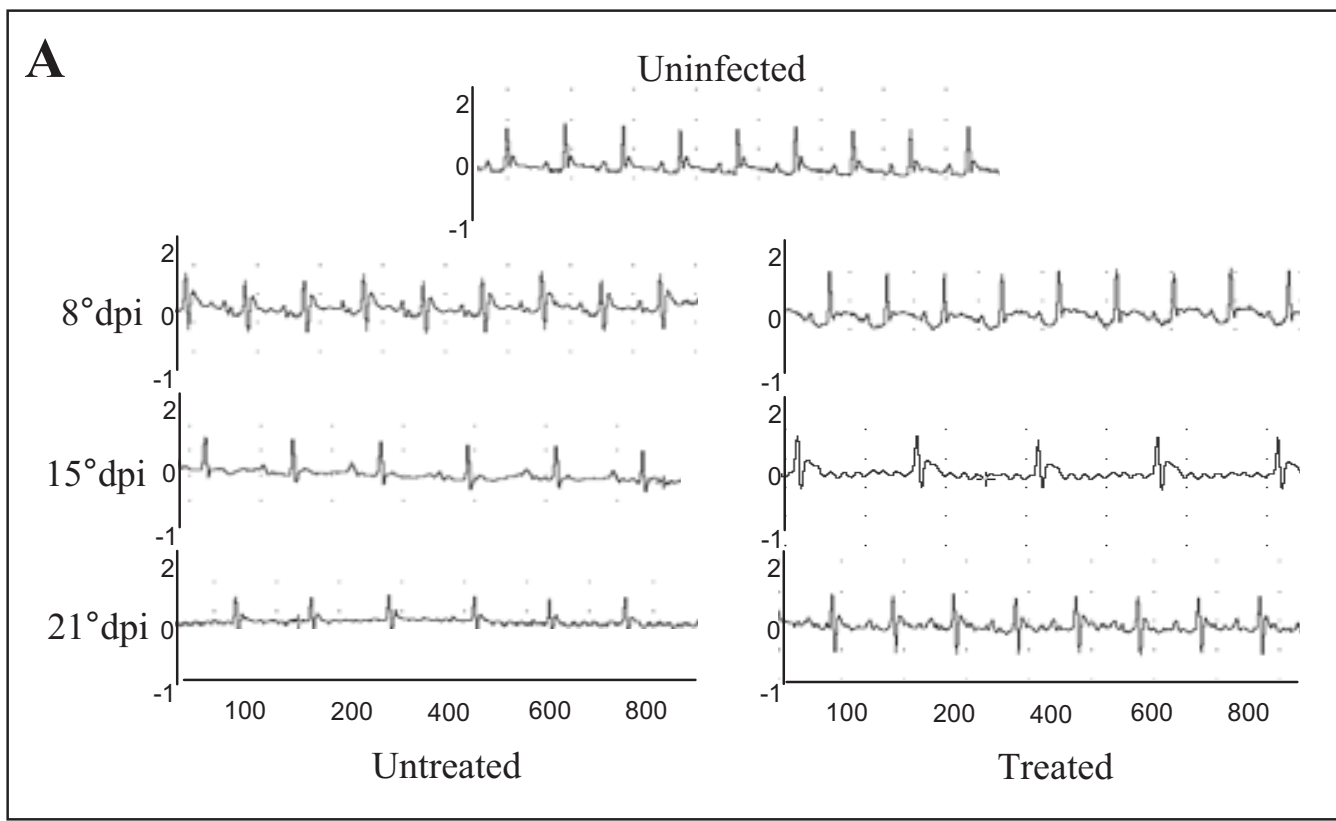

B

C
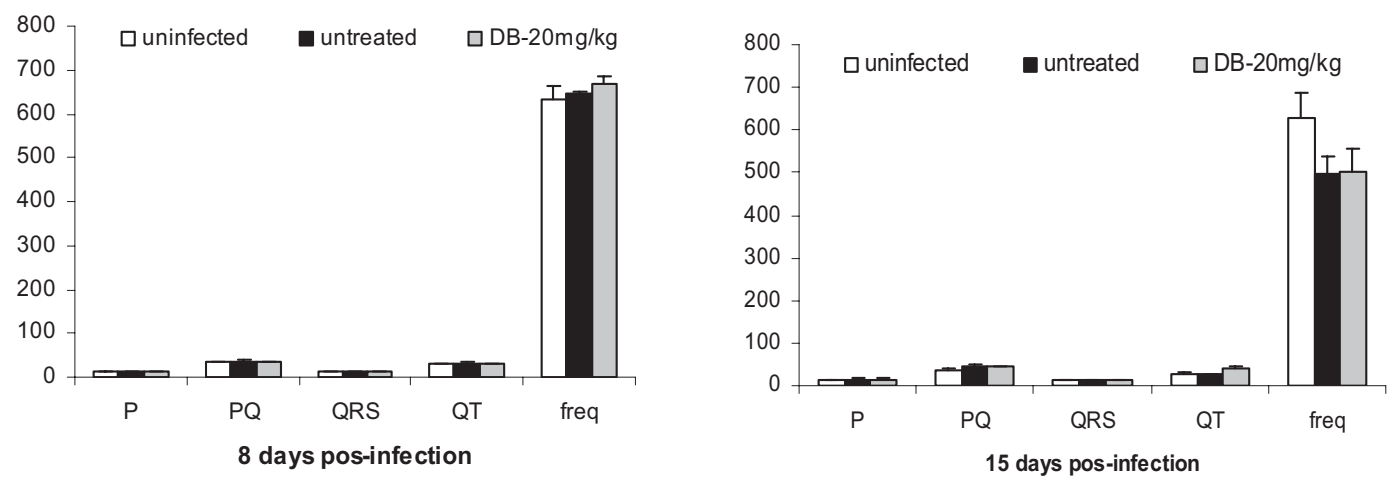

D

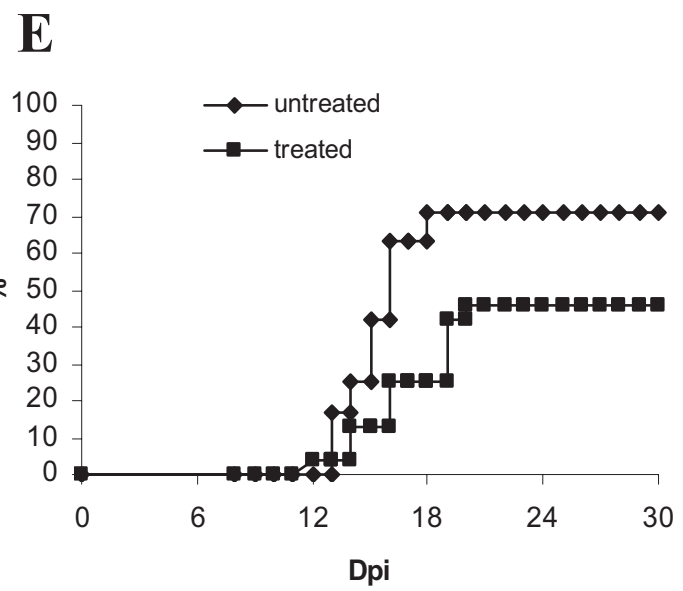

Figure 3. Electrocardiographic finding during acute phase of $T$. cruzi-infection, were verified by the variation at heart rate (beats/min) and P wave and PQ, QRS and QT intervals (ms) at uninfected (white), untreated (black) and DB-treated (gray) groups: (A) Electrocardiographic tracing at $8^{\circ} \mathrm{dpi}, 15^{\circ} \mathrm{dpi}$ and $21^{\circ} \mathrm{dpi}$ of all groups; Electrocardiogram values at $8^{\circ} \mathrm{dpi}(\mathbf{B}), 15^{\circ} \mathrm{dpi}(\mathrm{C})$ and at $21^{\circ} \mathrm{dpi}($ (D). Cumulative mortality curve of untreated $(\bullet)$ and DB-treated $(\mathbf{\square})$ T. cruzi-infected groups accompanied until $30 \mathrm{dpi}(\mathrm{E})$.

Asterisks indicate significant differences of DB569-treated in relation to the untreated group for electrocardiographic finding, $p \leq 0.01$. 

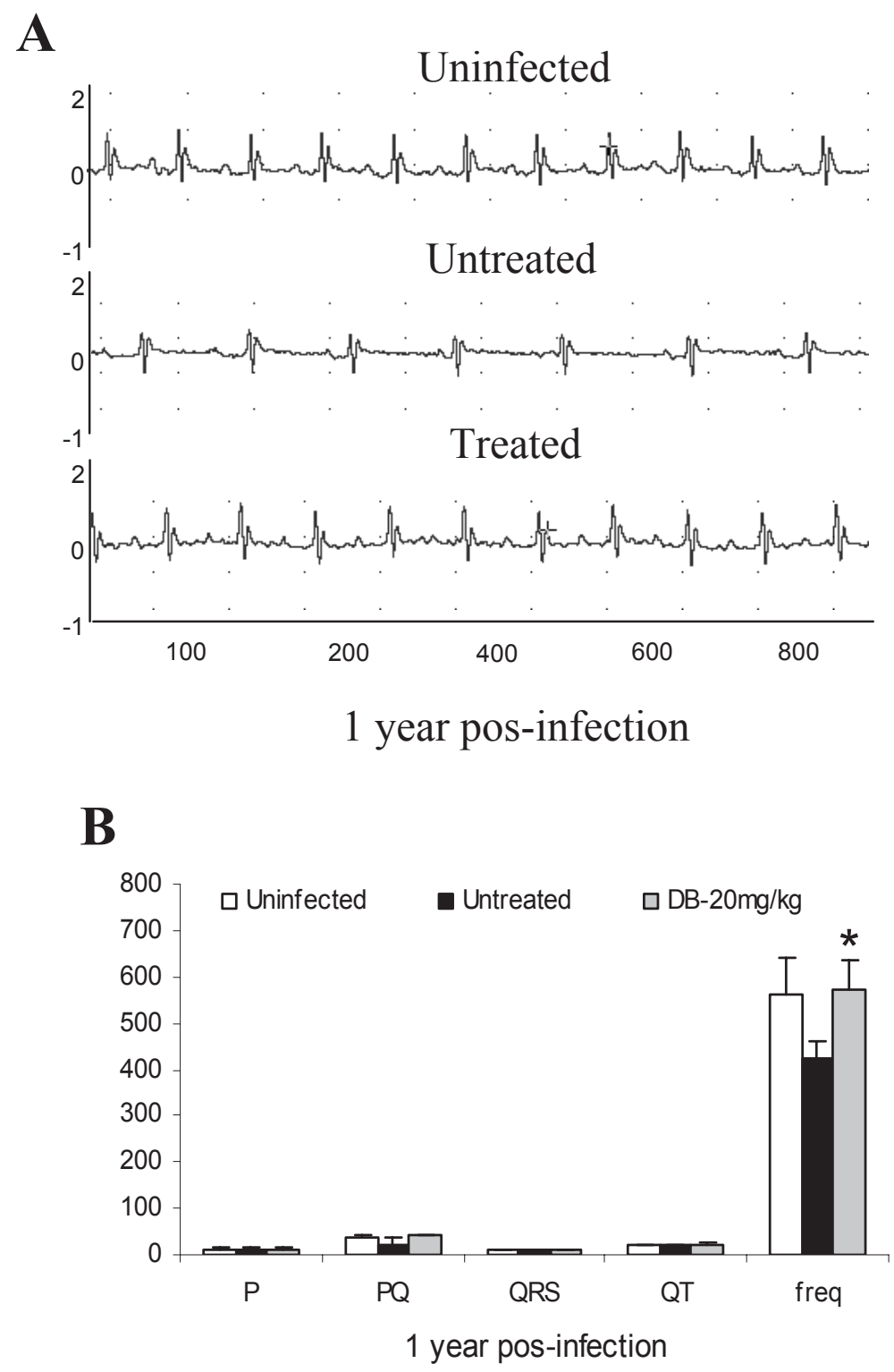

Figure 4. Electrocardiographic finding at chronic phase after one year of $T$. cruzi infection were verified by the variation at heart rate (beats/ $\mathrm{min}$ ) and $\mathrm{P}$ wave and $\mathrm{PQ}, \mathrm{QRS}$ and QT intervals $(\mathrm{ms})$ at uninfected (white), untreated (black) and DB-treated (gray) groups: (A) electrocardiographic tracing and (B) electrocardiographic parameters of all groups.

Asterisks indicate significant differences of DB569-treated in relation to the untreated group, $p \leq 0.01$.

DB569 in the development of ECG alterations in both acute and chronic murine T. cruzi infection.

As previously reported, our data confirmed that T. cruzi-infected mouse represents an interesting model for analyzing ECG alterations due to parasite infection, displaying electrocardiographic abnormalities (Rivarola et al. 2005; Zaidenberg et al. 2006). In our present experimental model, these abnormalities were mostly related to a remarkable decrease of the cardiac frequency, as also reported by others (Bustamante et al. 2005). Our results showed that DB569 considerably reduced the ECG alterations in both acutely and chronically $T$. cruzi-infected mice similarly as described in other studies performed with different drugs (Lo Presti et al. 2004; Rivarola et al. 2005; Garcia et al. 2005; Zaidenberg et al. 2006).

Interestingly, the DB569 protection was directly related to the levels of cardiac cytotoxic $\mathrm{T}$ cells, since the treatment also decreased the levels of $\mathrm{CD}^{+}$cells found in the inflamed heart and partially recovered the $\mathrm{CD} 4^{+} / \mathrm{CD}^{+}$ratio that was 0.37 and 0.50 in untreated mice and DB569 treated mice, respectively. This is an interesting finding since it 
has been reported that in acutely and chronically T. cruzi-infected patients and during the murine infection, cardiac $\mathrm{CD} 4^{+} / \mathrm{CD}^{+} \mathrm{T}$ cell ratio shows a predominance of $\mathrm{CD}^{+} \mathrm{T}$ cells suggestive of an immunologic imbalance response (Higuchi et al. 1997; Marino et al. 2004; Fuenmayor et al. 2005; Henriques-Pons et al. 2005; Cunha-Neto et al. 2006).

The parasitism control and decreased $\mathrm{CD} 8^{+} \mathrm{T}$ cells levels in the myocardium of DB569-treated group was concomitantly found with an unaltered ECG profile that might be ultimately contributing to their increased survival rates. This therapeutic effect of DB569 on the bradyarrhythmia could be related to trypanocidal effect of the diamidine and/or the modulation of the host immunological response to the parasite. In fact, the regulation of immune response in $T$. cruzi-infected mice by other trypanocidal drugs, such as benznidazole, has already been reported (Olivieri et al. 2006; Romanha et al. 2002), and our previous in vitro data showed that parasite elimination was more effective after treatment of infected phagocytic cells as compared to cardiac ones (De Souza et al. 2004). Additionally, as DB569 induces apoptosis in T. cruzi (De Souza et al. 2006b), the presence of apoptotic parasites in cardiac tissues could contribute for regulation of the host inflammatory orchestra (Fadok et al. 2000; DosReis et al. 2005).

Although the mechanisms leading to chronic chagasic cardiomyopathy (CCC) are still unknown, the initial cardiac damage caused by the parasite seems to play a critical role for the progression of the disease (Tarleton, 2001). Moreover, specific trypanocidal treatment seems to be associated with delay in human heart disease progression (Viotti et al. 2006). In addition, although accumulated data highlighted the concept that tissue damage is direct or indirectly induced by T. cruzi itself and thus the control of parasite load by a host effective inflammatory response could represent a prerequisite to arrest the evolution of the disease, an excessive or imbalanced inflammation (as an auto reactivity response) can also contribute to the genesis of the cardiac damage (reviewed in Dutra et al. 2005). In fact, chronic chagasic patients submitted to benznidazole therapy although not parasitologically cured, presented a marked reduction in the occurrence of ECG changes and a lower frequency of deterioration in their clinical conditions (Viotti et al. 1994).
Then, despite the genesis of CCC, the current concept suggests that parasite eradication is a requisite to arrest the evolution of the Chagas disease pointing to the urgent need for the discovery and development of safe and effective therapy for the chagasic patients. In this context, our results suggest that diamidines are a potential class of compounds to be employed further in vitro and in vivo studies for Chagas' disease treatment.

\section{Acknowledgments}

MNCS and EMDS would like to thank Conselho Nacional Desenvolvimento Científico e Tecnológico (CNPq); Fundação Carlos Chagas Filho de Amparo a Pesquisa do Estado do Rio de Janeiro (FAPERJ) and PAPES IV/FIOCRUZ for financial support. The authors thank Dr. Vinicius Cottade-Almeida (LBC/IOC/FIOCRUZ) for the careful revision. The authors express special appreciation to Dr. David Boykin (Department of Chemistry, Georgia State University, Atlanta, USA) for manuscript revision and for supplying the DB569.

\section{References}

Armenti, A. 2006. Long-term cardiac outcomes of treating chronic Chagas disease with benznidazole versus no treatment: a nonrandomized trial. Ann. Intern. Med., 144:724-34.

Bustamante, J.M., Rivarola, H.W. and Fretes, R. et al. 2005. Weekly electrocardiographic pattern in mice infected with two different Trypanosoma cruzi strains. Int. J. Cardiol., 102:211-7.

Bustamante, J.M., Rivarola, H.W. and Fernandez, A.R. et al. 2003. Trypanosoma cruzi reinfections provoke synergistic effect and cardiac beta-adrenergic receptors' dysfunction in the acute phase of experimental Chagas' disease. Exp. Parasitol., 103:136-42.

Coura, R.J. and De Castro, S.L. 2002. A critical review on Chagas' disease chemotherapy. Mem. Inst. Oswaldo. Cruz., 97:3-24.

Dávila-Spinetti, D.F., Colmenarez-Mendoza, H.J. and Lobo-Vielma, L. 2005. Mechanisms responsible for myocardial damage progression in Chronic Chagas disease. Rev. Esp. Cardiol., 58:1007-09.

De Souza, E.M., Lansiaux, A. and Bailly, C. et al. 2004. Phenyl substitution of furamidine markedly potentiates its anti-parasitic activity against Trypanosoma cruzi and Leishmania amazonensis. Biochem. Pharmacol., 68:593-600.

De Souza, E.M., Oliveira, G.M. and Boykin, D.W. et al. 2006a. Trypanocidal activity of the phenyl-substituted analogue of furamidine DB569 against Trypanosoma cruzi infection in vivo. J. Antimicrob Chemother, 58:610-4.

De Souza, E.M., Menna-Barreto, R. and Araujo-Jorge, T.C. et al. 2006b. Antiparasitic activity of aromatic diamidines is related to apoptosislike death in Trypanosoma cruzi. Parasitology, 133:75-9.

DosReis, G.A., Freire-de-Lima, C.G. and Nunes, M.P. et al. 2005. The importance of aberrant T-cell responses in Chagas disease. Trends Parasitol, 21:237-43.

Dutra, W.O., Rocha, M.O.C. and Teixeira, M.M. 2005. The clinical immunology of human Chagas disease. Trends in Parasitology, 21:581-7.

Cunha-Neto, E., Bilate, A.M. and Kenneth, V. et al. 2006. Induction of cardiac autoimmunity in Chagas heart disease: A case for molecular mimicry. Autoimmunity, 39:41-54. 
Fadok, V.A., Bratton, D.L. and Rose, D.M. et al. 2000. A receptor for phosphatidylserine-specific clearance of apoptotic cells. Nature, 405:85-90.

Fuenmayor, C., Higuchi, M.L. and Carrasco, H. et al. 2005. Acute Chagas' disease: immunohistochemical characteristics of T-cell infiltrate and its relationship with T. cruzi parasitic antigens. Acta. Cardiol., 60:33-7.

Garcia, S., Ramos, C.O. and Senra, J.F. et al. 2005. Treatment with benznidazole during the chronic phase of experimental Chagas' disease decreases cardiac alterations. Antimicrob Agents Chemother., 49:1521-8.

Golgher, D. and Gazzinelli, R.T. 2004. Innate and acquired immunity in the pathogenesis of Chagas disease. Autoimmunity, 37:399-409.

Henriques-Pons, A., Olivieri, B.P. and Oliveira, G.M. et al. 2005. Experimental infection with Trypanosoma cruzi increases the population of CD8(+), but not CD4(+), immunoglobulin G Fc receptor-positive T lymphocytes Infect. Immun., 73:5048-52.

Higuchi, M.D., Ries, M.M. and Aiello, V.D. et al. 1997. Association of an increase in CD8+ T cells with the presence of Trypanosoma cruzi antigens in chronic, human, chagasic myocarditis. Am. J. Trop. Med. Hyg., 56:485-9.

Lansiaux, A.F., Tanious, Z. and Mishal, L. et al. 2002. Distribution of furamidine analogues in tumor cells: targeting of the nucleus or mitochondria depending on the amidine substitution. Cancer Res., 62:7219-29.

Leon, J.S. and Engman, D.M. 2003. The significance of autoimmunity in the pathogenesis of Chagas heart disease. Front Biosci., 8:315-22.

Lo Presti, M.S., Rivarola, H.W. and Bustamante, J.M. et al. 2004. Thioridazine treatment prevents cardiopathy in Trypanosoma cruzi infected mice. Int. J. Antimicrob Agents, 23:634-6.

Maguire, J.H., Hoff, R. and Sherlock, I. et al. 1987. Cardiac morbidity and mortality due to Chagas' disease: prospective electrocardiographic study of a Brazilian community. Circulation, 75:1140-5.

Marin-Neto, J.A., Simoes, M.V. and Sarabanda, A.V. 1999. Chagas' heart disease. Arq. Bras. Cardiol., 72:247-80.

Marino, A.P., da Silva, A. and dos Santos, P. et al. 2004. Regulated on activation, normal $\mathrm{T}$ cell expressed and secreted (RANTES) antagonist (Met-RANTES) controls the early phase of Trypanosoma cruzielicited myocarditis Circulation, 110:1443-9.

Meirelles, M.N., Chiari, E. and de Souza, W. 1982. Interaction of bloodstream, tissue culture-derived and axenic culture-derived trypomastigotes of Trypanosoma cruzi with macrophages. Acta Trop, 39:195-203

Olivieri, B.P., Cotta-De-Almeida, V. and Araujo-Jorge, T. 2002. Benznidazole treatment following acute Trypanosoma cruzi infection triggers CD8+ T-cell expansion and promotes resistance to reinfection. Antimicrob Agents Chemother, 46:3790-6.

Olivieri, B.P., Farias-De-Oliveira, D.A. and Araujo-Jorge, T.C. et al. 2005. Benznidazole therapy in Trypanosoma cruzi-infected mice blocks thymic involution and apoptosis of $\mathrm{CD} 4+\mathrm{CD} 8+$ double-positive thymocytes. Antimicrob Agents Chemother, 49:1981-7.
Olivieri, B.P., de Souza, A.P. and Cotta-de-Almeida, V. et al. 2006. Trypanosoma cruzi: Alteration in the lymphoid compartments following interruption of infection by early acute benznidazole therapy in mice. Exp. Parasitol., 114:228-34.

Petkova, S.B., Huang, H. and Factor, S.M. et al. 2001. The role of endothelin in the pathogenesis of Chagas' disease. Int. J. Parasitol., 31: 499-511.

Rivarola, H.W., Bustamante, J.M. and Lo Presti, S. et al. 2005. Trypanosoma cruzi: chemotherapeutic effects of clomipramine in mice infected with an isolate obtained from an endemic area. Exp. Parasitol., 111:80-6.

Rocha, M.O., Ribeiro, A.L. and Teixeira, M.M. 2003. Clinical management of chronic Chagas cardiomyopathy. Front Biosci., 8:44-54.

Romanha, A.J., Alves, R.O. and Murta, S.M. et al. 2002. Experimental chemotherapy against Trypanosoma cruzi infection: essential role of endogenous interferon-gamma in mediating parasitologic cure. $J$. Infect. Dis., 186:823-8.

Salles, G., Xavier, S. and Sousa, A. et al. 2003. Prognostic value of QT interval parameters for mortality risk stratification in Chagas' disease: results of a long-term follow-up study. Circulation, 108:305-12.

Soeiro, M.N., De Souza, E.M. and Stephens, C.E. et al. 2005. Aromatic diamidines as antiparasitic agents. Expert Opin. Investig. Drugs, 14:957-72.

Tarleton, R.L. 2001. Parasite persistence in the aetiology of Chagas disease Int. J. Parasitol., 31:550-4.

Viotti, R., Vigliano, C. and Armenti, H. et al. 1994.Treatment of chronic Chagas' disease with benznidazole: clinical and serologic evolution of patients with long-term follow-up. Am. Heart J., 127:151-62.

Viotti, R., Vigliano, C. and Lococo, B. et al. 2006. Long-term cardiac outcomes of treating chronic Chagas disease with benznidazole versus no treatment: a nonrandomized trial. Ann. Intern. Med., 144:724-34.

Werbovetz, K. 2006. Diamidines as antitrypanosomal, antileishmanial and antimalarial agents. Curr. Opin. Investig. Drugs., 7:147-57.

Wilson, W.D., Nguyen, B. and Tanious, F.A. et al. 2005. Dications that target the DNA minor groove: compound design and preparation, DNA interactions, cellular distribution and biological activity. Curr. Med. Chem. Anti. Cancer Agents, 5:389-408.

World Health Organization. 2002. Control of Chagas disease. WHO Technical Report Series 905:109.

Zaidenberg, A., Luong, T. and Lirussi, D. et al. 2006. Treatment of experimental chronic Chagas disease with trifluralin. Basic Clin. Pharmacol. Toxicol., 98:351-6. 